\title{
Predicting cell-type-specific non-coding RNA transcription from
}

\section{genome sequence}

Masaru Koido ${ }^{1,2}$, Chung-Chau $\mathrm{Hon}^{3}$, Satoshi Koyama ${ }^{4}$, Hideya Kawaji ${ }^{5}$, Yasuhiro Murakawa $^{6}$, Kazuyoshi Ishigaki ${ }^{1,7,8,9}$, Kaoru Ito ${ }^{4}$, Jun Sese ${ }^{10,11}$, Yoichiro Kamatani ${ }^{1,12}$,

5 Piero Carninci ${ }^{13,14},{ }^{*}$ Chikashi Terao ${ }^{1,15,16,17}$

1. Laboratory for Statistical and Translational Genetics, RIKEN Center for Integrative Medical Sciences, Yokohama, 230-0045, Japan.

2. Division of Molecular Pathology, Department of Cancer Biology, Institute of Medical Science, The University of Tokyo, Tokyo, 108-8639, Japan.

10 3. Laboratory for Genome Information Analysis, RIKEN Center for Integrative Medical Sciences, Yokohama, 230-0045, Japan.

4. Laboratory for Cardiovascular Genomics and Informatics, RIKEN Center for Integrative Medical Sciences, Yokohama, 230-0045, Japan.

5. Preventive Medicine and Applied Genomics Unit, RIKEN Center for Integrative Medical Sciences, 15 Yokohama, 230-0045, Japan.

6. RIKEN-IFOM Joint Laboratory for Cancer Genomics, RIKEN Center for Integrative Medical Sciences, Yokohama, 230-0045, Japan.

7. Divisions of Genetics and Rheumatology, Department of Medicine, Brigham and Women's Hospital, Harvard Medical School, Boston, MA 02115, USA.

20 8. Center for Data Sciences, Harvard Medical School, Boston, MA 02115, USA.

9. Program in Medical and Population Genetics, Broad Institute of MIT and Harvard, Cambridge, MA 02142 , USA.

10. Artificial Intelligence Research Center, National Institute of Advanced Industrial Science and Technology, Aomi, Koto-ku, Tokyo, 135-0064, Japan.

25 11. Humanome Lab Inc., Tokyo, 104-0045, Japan.

12. Laboratory of Complex Trait Genomics, Department of Computational Biology and Medical Sciences, Graduate School of Frontier Sciences, The University of Tokyo, Tokyo, 108-8639, Japan.

13. Laboratory for Transcriptome Technology, RIKEN Center for Integrative Medical Sciences, Yokohama, 230-0045, Japan.

30 14. Laboratory for Single Cell Technologies, RIKEN Center for Integrative Medical Sciences, Yokohama, 230-0045, Japan. 
bioRxiv preprint doi: https://doi.org/10.1101/2020.03.29.011205; this version posted March 31, 2020. The copyright holder for this preprint (which was not certified by peer review) is the author/funder. All rights reserved. No reuse allowed without permission.

15. Clinical Research Center, Shizuoka General Hospital, Shizuoka, 420-8527, Japan.

16. The Department of Applied Genetics, The School of Pharmaceutical Sciences, University of Shizuoka, Shizuoka, 422-8526, Japan.

17. Lead Contact.

$5 \quad{ }^{*}$ Correspondence: chikashi.terao@riken.jp 


\section{SUMMARY}

Transcription is regulated through complex mechanisms involving non-coding RNAs (ncRNAs).

However, because transcription of ncRNAs, especially enhancer RNAs, is often low and cell

type-specific, its dependency on genotype remains largely unexplored. Here, we developed

5 mutation effect prediction on ncRNA transcription (MENTR), a quantitative machine learning

framework reliably connecting genetic associations with expression of ncRNAs, resolved to the

level of cell type. MENTR-predicted mutation effects on ncRNA transcription were concordant

with estimates from previous genetic studies in a cell type-dependent manner. We inferred

reliable causal variants from 41,223 GWAS variants, and proposed 7,775 enhancers and 3,548

10 long-ncRNAs as complex trait-associated ncRNAs in 348 major human primary cells and tissues,

including plausible enhancer-mediated functional alterations in single-variant resolution in

Crohn's disease. In summary, we present new resources for discovering causal variants, the

biological mechanisms driving complex traits, and the sequence-dependency of ncRNA

regulation in relevant cell types. (145/150 words) 


\section{INTRODUCTION}

Large scale human transcriptome analyses have revealed that long-ncRNA (IncRNA) and enhancer RNA expression is tissue- and cell-type-specific and regulates human development and homeostasis ((DGT) et al., 2014; Andersson et al., 2014; Hon

5 et al., 2017). Genome-wide association studies (GWAS) have found many complex traits-associated variants enriched in loci from which ncRNAs are transcribed (Andersson et al., 2014; Boyd et al., 2018; Hon et al., 2017; Kristjánsdóttir et al., 2018; Maurano et al., 2012) as well as substantial heritability enrichments in transcribed enhancer regions (Finucane et al., 2015). These findings implicate ncRNAs in the

10 mechanisms driving complex traits. GWAS has allowed inference of trait-relevant tissues or cell-types (Finucane et al., 2015, 2018), biological pathways (lotchkova et al., 2019; Lamparter et al., 2016), and therapeutic drugs (Terao et al., 2016); therefore, determining the influence of non-coding causal variants from GWAS on ncRNA expression in relevant cell-types is a promising approach to deepen our understanding

15 of complex trait mechanisms (Figure 1A). For mRNAs, such analyses have already improved understanding of the genetic architecture of complex traits; mRNA expression quantitative trait loci (eQTL) information, resolved to the tissue- or cell-type- level, has been linked to GWAS results to implicate the cell types and tissues involved in complex 
traits (Ardlie et al., 2015; Ishigaki et al., 2017). However, mapping eQTL in a given tissue or cell-type has traditionally required gene expression datasets for the target tissue or cell-type, as well as genotypes from, in general, over 100 individuals (Figure S1A). Discovery of eQTL for ncRNAs is especially challenging due to their often low 5 expression levels and high cell-type-specificity (Andersson et al., 2014; Hirabayashi et al., 2019; Hon et al., 2017).

Recently, machine learning (ML) models, including convolutional neural networks (CNN), have succeeded in predicting epigenetic events (Hoffman et al., 2019; Zhou and Troyanskaya, 2015) and gene expression levels in a tissue- or

10 cell-type-dependent manner (Kelley et al., 2018; Zhou et al., 2018). This advance has been made by extending conventional, short-range motif analysis and performing $\mathrm{ML}$ on kilobase (kb) scale genome sequence patterns. Despite training ML models without individual genotype data (in other words, models assume that the training data comes from cells with the reference genome sequence), in silico mutagenesis can predict the 15 effect of genetic variants on a particular transcript. Estimates of mutation effect made based on ML have been comparable with those based on QTL studies for mRNA expression (Kelley et al., 2018; Zhou et al., 2018). Notably, in silico mutagenesis has achieved comparable performance despite not requiring genotype data, thus 
accumulated transcriptome datasets may be suitable for training. Furthermore, in silico predictions are not affected by linkage disequilibrium (LD), allowing pin-point prediction of causal variants on mRNA transcriptional changes (Zhou et al., 2018). These previous studies motivated us to expand the ML-based framework to cell-type-specific

expression of ncRNA (Figure 1A, Figure S2A). Here we demonstrate MENTR (mutation effect prediction on $\underline{n}$ CRNA transcription), a ML program trained using cell-type-specific ncRNA and enhancer transcription, measured by cap analysis of gene expression (CAGE), that can accurately predict the effect of mutations on ncRNA expression ((DGT) et al., 2014; Andersson et al., 2014).

\section{RESULTS}

\section{Strategy to predict mutations' effects on ncRNAs}

MENTR ML models learn cell-type-specific transcription of promoters (including $>20 \mathrm{~K}$ types of IncRNA) and $>65 \mathrm{~K}$ types of enhancers from only $+/-100-\mathrm{kb}$ human

reference genome sequence (hg19) surrounding transcriptional start site (TSS) (Figure 1B and $1 \mathrm{C})$. We developed MENTR ML models by combining deep convolutional neural networks (from 2-kb sequence bin to 2002 epigenetic features, using publicly available pre-trained models (Zhou and Troyanskaya, 2015; Zhou et al., 2018)) and binary 
classifiers using non-linear, gradient boosting trees (from the many epigenetic features in +/- 100-kb sequence to accurate transcription probability; see METHOD DETAILS) (Chen and Guestrin, 2016). The binary classifier outputs a probability of expression for each tissue or cell-type, chosen because we focus here on predicting lowly-expressed

5 RNAs whose quantitative measurement might be not reliable (Hirabayashi et al., 2019)).

We trained the MENTR ML models using the autosomal mRNA and ncRNA promoterand enhancer-level transcripts profiled by CAGE except for chromosome 8 and tested the accuracy using those of chromosome 8 (Figure S1B). After training, MENTR can predict mutation effects on transcripts (for each tissue or cell-type used for training) by

10 in silico mutagenesis, the estimates of which are prioritizable based on the degree of probability change (Figure 1D) and are free from LD structure (Figure 1E).

\section{Accurate prediction of cell-type-specific promoter- and enhancer-level}

\section{transcription}

from 347 types of samples comprising a variety of primary cells and tissues (see METHOD DETAILS). Training MENTR using +/- 100-kb flanking the transcriptional start site (TSS) achieved the highest accuracy, and shorter input sequences slightly 
decreased accuracy (Figure S3). Interestingly, training based on only promoter expression could predict enhancer RNA expression, and vice versa, supporting that promoters and enhancers at least partially share sequence-based regulatory machineries. Training using only enhancer data slightly decreased predictive accuracy

5 for both promoters and enhancers, presumably due to technical limitation of accurate detection of enhancer RNAs. Thus, we decided to train using both promoters and enhancers, thereby including RNAs expressed over a wide range of abundances. To reduce computational costs, we used linear penalized logistic regression models using boosting (MENTR linear $_{\text {) }}$ (Bühlmann, 2006; Chen and Guestrin, 2016) as a binary

10 classifier in the above screening procedures.

MENTR revealed good prediction accuracy for enhancer expression in a probability-dependent manner (Figure 2A). The area under the receiver operating characteristic curve (AUROC) was $0.69 \pm 0.05$ for enhancers in 347 sample ontologies, $0.76 \pm 0.04$ for IncRNAs. Unsurprisingly, AUROC values for coding mRNA (0.83 \pm 0.02$)$

15 were higher than those for ncRNA, and predictions of small RNA, pseudogene, and short ncRNA expression were not always accurate (Figure 2B, Table S1, and Table S2). Even though MENTR was trained using expression as an on-off variable, the correlation between predicted expression probability and measured expression levels in FANTOM5 
CAGE transcriptome datasets was much higher than the previous method ExPecto (Zhou et al., 2018) for IncRNA (Wilcoxon signed rank test $\left.P=1.8 \times 10^{-58}\right)$ and $\mathrm{mRNA}(\mathrm{P}=$ $1.3 \times 10^{-58}$ ) (Figure S2B). ExPecto uses transcription strand information and thus cannot be used to predict bidirectionally-transcribed enhancer RNAs (see METHOD DETAILS).

5 These data indicated that MENTR was suitable for accurate prediction of lowly-expressed enhancers and IncRNAs, not only mRNAs, in CAGE transcriptomes.

Interestingly, prediction of transcription of promoters annotated as "CpG-less" was accurate (AUROC: $0.73 \pm 0.02$ ) but underperformed those annotated as containing CpGs (Wilcoxon-Mann-Whitney test $\mathrm{P}=2.3 \times 10^{-178}$ ) (Figure S4). This suggests that

10 MENTR ML models have learned the importance of $\mathrm{CpG}$ sites for transcription without any prior knowledge, but also learned the exception rules (transcription from CpG-less sites) at the same time. Moreover, transcription of promoters annotated as "TATA-less" were rather more predictable than those annotated as including TATA boxes (Figure S4; $\left.P=2.2 \times 10^{-31}\right)$. Recent studies have shown the existence of many TATA-less promoters

15 in mammals, with transcription initiating in a different and more predictably flanking-sequence-dependent manner compared to TATA-containing promoters (Anish et al., 2009; Donczew and Hahn, 2017). Taken together, MENTR could learn genome sequence patterns that influence transcription. Therefore, interpreting the output of 
MENTR might be useful for understanding the biology of gene expression.

Considering the quite low expression levels of many ncRNAs, we were concerned that MENTR could learn anomalous read patterns, noise, or artifacts related to transcript mappability that vary depending on sequence context, rather than

5 reproducible transcriptional status; if this were the case, it would be unexpected if MENTR could accurately predict the expression patterns of RNAs that are in fact transcribed, but are not detected due to low depth of sequencing or degradation. To evaluate this possibility, we took advantage of enhancer RNA transcription data in five ENCODE cell lines profiled using NET-CAGE, a sensitive method for measuring

10 nascent RNA transcription (Hirabayashi et al., 2019). We then evaluated false positive (FP) predictions, in which a transcript was not detected by the standard CAGE method, but MENTR predicted a probability of expression $>0.5$ (based on ML models trained using standard CAGE data). We observed that $31-70 \%$ of the transcripts which were initially considered as FP predictions were actually transcribed across all five cell lines

15 when assayed using NET-CAGE (Table S3; $\mathrm{P}<3.6 \times 10^{-3}$ ). These results indicate that MENTR ML models accurately learned sequence-dependent transcription patterns for enhancers, even though a substantial fraction of them might have been undetectable in the standard CAGE transcriptomes used for training. This indicates that we might 
underestimate the accuracy of MENTR, especially for enhancers (and possibly small

RNAs, pseudogenes, and short ncRNA) by AUROC values (Figure 2, Table S1, and

Table S2) using standard CAGE data to define ground truth. Thus, we did not filter out

MENTR ML models by their AUROC values in the following analyses.

Verification of predicted mutation effects by comparing with previous

eQTL/caQTL studies

Having trained MENTR to predict RNA transcription based on genomic sequence, we next sought to evaluate its ability to predict the effect of mutations on

10 transcription. In order to verify the accuracy of predictions based on in silico mutagenesis, we additionally trained a MENTR ML model on CAGE transcriptomes of lymphoblastoid cell lines (LCL) (AUROC for promoter $=0.82$ and that for enhancer $=$ 0.71; Table S4) for which eQTL, as well as chromatin accessibility QTL (caQTL), were available (Garieri et al., 2017; Kumasaka et al., 2019). We compared in silico mutation

15 effects predicted by MENTR and the effect sizes in the two previous QTL studies for the alternative alleles.

First, we compared in silico mutation effects from MENTR ML models trained on the 347 FANTOM5 CAGE transcriptomes with eQTL coefficients in LCL on promoter- 
and enhancer-level CAGE transcriptome analysis (Garieri et al., 2017) (Figure S5A).

We found that the higher the predicted mutation effects by the MENTR ML model trained using LCL data, the more consistent with the observed effect of the variant in the eQTL study. This was true even for enhancers and IncRNAs (Figure 3A-C and Figure

5 S6A; see red line). While the mutation effects predicted in the LCL model did not always provide the best accuracy, the models giving good accuracy were trained on transcriptional profiles highly correlated with that of LCL, including lymphocyte in FANTOM5 (Figure 3A-C and Figure S6A; see orange lines in heatmap colors). Prediction accuracy among the different models were variable, especially for low

10 abundance transcripts. Bad prediction in models trained using cells or tissues with transcription profiles dissimilar to LCLs was obvious for enhancer and IncRNA (Figure 3A-C; see blue lines in heatmap colors). These results indicated that cell-type-specific training is important for accurate prediction of mutation effects, especially for enhancer and IncRNA. Second, we compared in silico mutation effects with coefficients of caQTL (Kumasaka et al., 2019) under the hypothesis that transcription of CAGE promoter- and enhancer-regions were regulated similarly to chromatin accessibility, as measured by ATAC-seq peaks (Figure S5B). Testing on the variant-promoter and variant-enhancer 
pairs supports the same conclusion as the analysis using eQTL (Figure 3D-F, Figure S6B), indicating that prediction accuracy of MENTR is not dependent on the CAGE method and that chromatin accessibility is regulated in a sequence-dependent manner similar to that of transcription of enhancers and promotors. Although our predictions

5 were based on binary transcription state, the in silico mutation effect sizes were correlated with the effect size in the caQTL study for both promoters (Spearman's $\rho=$ 0.25 [95\% Cl: $0.24-0.26])$ and enhancers (Spearman's $\rho=0.36$ [95\% Cl: 0.32-0.36]) (Figure S7). Taken together, this shows that MENTR accurately predicts transcription, including enhancers, in a cell-type-dependent manner, especially when we used

10 predictions with higher mutation effect size. Hereafter, we defined the permissive threshold achieving $~ 80 \%$ concordance and the robust threshold achieving $>90 \%$ concordance in Figure $3 \mathrm{~A}-\mathrm{C}$ as 0.05 and 0.1 of absolute in silico mutation effects, respectively.

Next, we analyzed 26 types of tissues assayed by CAGE in FANTOM5 and

15 also in GTEx (manually matched; Table S5) and compared results between in silico mutagenesis by MENTR and GTEx eQTL. We limited this analysis to variants within 1-kb of transcripts, because almost all non-zero mutation effects were obtained +/- 1-kb from TSS (for example, 19,376 out of 86,724 variant-promoter pairs showed non-zero 
mutation effects, from which 18,226 pairs (94.0\%) were in +/- 1-kb from TSS (Table S6)). We aggregated the promoter-level predictions of mutation effects into gene-level predictions (Figure S8A) and, by comparing with GTEx eQTL estimates, verified that MENTR's prediction, at both permissive and robust thresholds, were accurate in almost

5 all types of tissues (Figure S8B; Wilcoxon signed rank test $P=9.8 \times 10^{-7}$ for permissive threshold and $P=4.2 \times 10^{-7}$ for robust threshold). These results strongly support the applicability of in silico mutagenesis using MENTR to predict the effect of genetic variants on transcription in many types of tissues.

Seeking additional evidence to support the predictive validity of MENTR, we

10 analyzed variants whose effects on mRNA transcription had been tested using reporter assays (Zhou et al., 2018). We predicted in silico mutation effects of the rs 147398495 (chr3:46249943 GTTC>G; CCR1 locus) and rs381218 (chr6:32977420:G>T; HLA-DOA locus) and the variants in LD $\left(r^{2} \geq 0.2\right.$ in 1 KGp3v5 EUR) on the representative promoter. Interestingly, the variants with the strongest in silico mutation effects were the lead 15 variants themselves (rs147398495 and rs381218) as supported by experimental evidence from the previous study (Zhou et al., 2018). Importantly these were also the only variants satisfying the robust threshold of in silico mutation effect (Figure S9). 


\section{Cataloging and prioritizing GWAS findings by MENTR}

We evaluated in silico mutation effects of previously reported GWAS variants

(in GWAS catalog and our previous studies; Akiyama et al., 2017; Ishigaki et al., 2019;

Kanai et al., 2018) as well as variants in LD with the variants $\left(r^{2} \geq 0.7\right.$, see METHOD

5 DETAILS) to facilitate interpretation of GWAS results that may alter ncRNA transcription.

As a result, we identified over ten thousand (permissive threshold) or $\sim 500$ (robust

threshold) ncRNAs associated with GWAS traits (Table1 and Table S7-S9). We

released all these results (GWAS trait-associated ncRNA database) as publicly

available resources in a user-friendly GUI application for both Windows and Mac users

10 (url). (will do after acceptance)

Among 42 complex diseases catalogued in Biobank Japan, 36 diseases

showed positive correlations between effect sizes of risk variants and their in silico

mutation effects (after conditioning on MAF, transcription type (promoter or enhancer)

and distance between variants and transcripts). Among them, 15 diseases, including

15 rheumatoid arthritis (RA), Graves' disease (GD), chronic hepatitis B (CHB), and

pancreatic cancer (PaCa), showed significant correlations (Bonferroni-corrected level of significance $(P<0.05 / 42))$ (Figure 4 and Table S10). This indicates alteration of ncRNA expression by the variants that underlies risk for these complex diseases. 
We further found that the MENTR could suggest plausible functional roles of GWAS variants for complex traits. We evaluated mutation effects of $95 \%$ credible sets of over 4000 causal variants in 139 independent loci from the fine-mapping study on inflammatory bowel disease (IBD) (Huang et al., 2017). Surprisingly, we found that the

5 credible sets in 117 loci (84\%) included variants with an effect on transcription predicted by in silico mutagenesis by MENTR (Figure 4B). In contrast, MENTR generally predicted no effect for $>70 \%$ of non-eQTL variant-transcript pairs near GTEx eQTL (Table S6). In these predictions, effects on transcription of enhancer and mRNA explained 96 and 86 loci, respectively (Figure 4C), reaffirming the importance of 10 transcribed enhancers in the etiology of complex traits.

Among the robust predictions of IBD credible sets (Table S11), MENTR pinpointed the best candidate of causal variants whose effects on transcriptional activity were experimentally validated in previous studies. In one example, rs17293632 in the SMAD3 region showed the highest posterior probability (40\%) in fine-mapping study of

15 Crohn's disease (CD) and is also known to be associated with risk of asthma and coronary artery disease (CAD) (Demenais et al., 2018; Huang et al., 2017; Turner et al., 2016) (Figure 5A), providing interpretation of the association in the context of cell-type-specific ncRNA transcription profiles. There are many variants showing strong 
eQTL effects on SMAD3 near rs17293632 (Figure 5B) making it difficult to identify a causal variant based on eQTL associations, and the LD structure of this region further complicates biological interpretation. On the other hand, MENTR predicted that the T allele of rs17293632 (a risk allele for Crohn's disease and asthma, and a protective

5 allele for CAD) decreased expression of the enhancer ADDG15067442347.E, in many types of cells plausibly relevant to these diseases: colon, neutrophil, natural killer cell, eosinophil, and macrophage (Fahy, 2009; Wéra et al., 2016; Yadav et al., 2011) (Figure 5C and Table S12). Consistent with this prediction, rs17293632 is located at an active enhancer region (a H3K27ac ChIP-seq peak) of many types of cells including human

10 sigmoid colon, rectal mucosa, monocytes, coronary artery smooth muscle cells. The T allele is known to lower chromatin accessibility and decrease binding of AP-1 transcription factor (Farh et al., 2015; Huang et al., 2017; Miller et al., 2016; Turner et al., 2016), supporting enhancer-mediated transcriptional regulation by this variant. Notably, MENTR predicted that $r 17293632$ and variants in LD $\left(r^{2} \geq 0.2\right.$ in $1 \mathrm{KGp3v5}$ EUR) would 15 have no direct effects on transcription from SMAD3's representative promoter (Figure 5C). rs17293632 was predicted to affect transcription from three minor promoters (p6, p11, p13@SMAD3) in corneal epithelial cell and CD14 ${ }^{+}$CD16 ${ }^{+}$monocyte (Table S11), suggesting that the alternative promoter usage might be regulated by the variant. 
Similarly, we found two additional plausible examples from the IBD credible sets. First, MENTR predicted that rs713875 (near LIF and HORMAD2 loci) lowered the expression of enhancer ADDG22030591785.E in CD-relevant CD8 ${ }^{+} \alpha-\beta$ T cell (Kadivar et al., 2016) (Figure S10 and Table S11), whose alternative allele was methylated in whole blood

5 (Hutchinson et al., 2014). Second, MENTR predicted that the alternative allele of rs4456788 (near ICOSLG loci) lowered the expression of enhancer ADDG21045616099.E in CD-relevant cell types such as CD4 ${ }^{+} \alpha-\beta$ T cell (Imam et al., 2018) (Figure S10 and Table S11), whose alternative allele decreased reporter activity of surrounding sequence in a cell line model (Mattioli et al., 2019). MENTR can thus

10 help prioritize plausible causative variants evidenced by previous findings and raise meaningful new hypotheses based on GWAS results.

\section{DISCUSSION}

We developed MENTR to predict the effect of genetic variants on transcription,

15 including transcription of ncRNAs. We demonstrated that in silico mutations predicted to have strong effects were highly concordant with the observed effects of known variants in a cell-type-dependent manner. As an example of how MENTR can facilitate interpretation of GWAS results and raise new mechanistic hypotheses, we pinpoint 
variants previously short-listed as credible causative variants and suggest plausible enhancer-mediated functional interpretations. In addition to the pre-calculated mutation effects of GWAS variants in CAGE transcriptomes from >300 types of human primary cells and tissues, we release programs which will allow others to predict the effect of

5 any variant, in any region of interest, on ncRNA as well as mRNA transcription (will available after acceptance).

An important feature of MENTR compared with previous relevant ML methods is the successful expansion of in silico mutagenesis principle into transcribed ncRNA, especially enhancers, which are bidirectionally transcribed from active enhancer

10 regions and enrich GWAS variants in a pathologically relevant cell types (Andersson et al., 2014; Kim et al., 2010; Murakawa et al., 2016). So far, 65K transcribed enhancers are known; but their expression has not been used for training ML models (Kelley et al., 2018; Zhou et al., 2018) despite their known high cell-specificity and trait relevance (Andersson et al., 2014; Hon et al., 2017). MENTR was trained using binarized RNA 15 transcription patterns, yet surprisingly our approach provided much more quantitative predictions than the previous method which learned expression levels along a quantitative spectrum (Figure S2).

Recently, the clinical importance of enhancers have been widely recognized, as 
shown in IBD (Boyd et al., 2018) and cancer studies (Zhang et al., 2019). Nevertheless, the functional roles of variants on or near enhancers are unclear, and only a few enhancer RNA QTL studies in LCL using limited number of subjects (154 European (Garieri et al., 2017) and 69 Yoruban (Kristjánsdóttir et al., 2018)) have been conducted.

5 Although enhancer RNA QTL might be partly discovered from ChIP-seq for histone marks (e.g., H3K4me1 and H3K27ac), DNase-seq or ATAC-seq, these studies have also been conducted for LCL, blood cells, and other limited types of cells using only hundreds of European or Yoruba individuals (Alasoo et al., 2018; Banovich et al., 2018; Bryois et al., 2018; Chen et al., 2016; Degner et al., 2012; Delaneau et al., 2019; Gate

10 et al., 2018; Kumasaka et al., 2019; Pelikan et al., 2018). An alternative approach is the experimental identification of transcribed enhancers (van Arensbergen et al., 2019), but it would still be challenging to conduct such experiments in many types of cells. Thus, the present study provides the only resource for interpreting the genetic regulation of enhancer transcription in $>300$ types of primary cells and tissues.

500 , depends heavily on tissue accessibility, and over $80 \%$ of donors are European (GTEx v7) (Aguet et al., 2017). Therefore, the effects of low-frequency or rare variants remain unknown, along with population-specific variants in non-Europeans. 
Furthermore, eQTL strongly depends on LD structure in the tested population. On the other hand, ML-based in silico mutagenesis trained using the human reference genome sequence is free from allele frequency and LD issues and instead prioritizes causal variants at single basepair resolution (Zhou et al., 2018). MENTR-predicted mutation

5 effects on transcription are zero in many cases, but provided pinpoint estimation of causal variants without depending on LD structure (Figure 4-5, and Table S6). MENTR is thus a useful new tool to filter GWAS variants in LD to identify the most likely causal variants.

Planned large scale whole-genome sequencing projects and increasing GWAS

10 samples sizes (Saunders et al., 2019) are expected to increasingly reveal infrequent and rare variants associated with complex traits. These variants will increasingly not be contained in eQTL catalogs thus other tools are needed to filter credible variants and make testable hypotheses about the mechanisms by which these variants drive disease, or other phenotypes of interest. MENTR is an attractive first choice to provide clues and 15 interpretations of such non-coding GWAS associations, especially for low-frequency variants. 


\section{Acknowledgments}

We deeply thank Dr. Nicholas Parrish for critically reviewing and editing the manuscript.

We thank FANTOM consortium members for providing datasets and valuable discussions. Computational resource of Al Bridging Cloud Infrastructure $(A B C l)$

5 provided by the National Institute of Advanced Industrial Science and Technology (AIST) was used for in silico mutagenesis.

\section{Author Contributions}

M.K, C-C.H, Y.K and C.T conceived the study. M.K conducted analysis with the help of

10 C-C.H, S.K, K.Ishigaki, K. Ito and C.P. C-C.H analyzed CAGE transcriptome data. H.K and Y.M analyzed NET-CAGE transcriptome data. M.K and C.T wrote the manuscript. J.S contributed to providing GPU computational resources which were necessary for the present study. P.C and C.T supervised this study.

\section{Declaration of Interests}

The authors declare no competing interests. 
Figure titles and legends

Figure 1. Strategy to find variants' effects on ncRNA transcription by MENTR.

(A) MENTR accurately predict effects of any mutation on ncRNA transcription in a

5 cell-type dependent manner, enabling interpretation of complex trait-associated loci.

Active enhancers, bidirectionally transcribed in a cell-type dependent manner, were shown as representative example of ncRNA. (B) Available transcriptome datasets for training MENTR ML models. CAGE transcriptome datasets other than those collected by FANTOM5 can be used. (C) MENTR ML models learn promoter and enhancer-level

10 on-off patterns from the TSS $\pm 100-\mathrm{kb}$ genome sequence. After the training ML models, mutation effects of any mutations on proximal promoters and enhancers can be inferred by comparing predicted expression probability from different input genome sequences (in silico mutagenesis). (D) Robust and permissive MENTR predictions can be selected by the threshold determined by comparison with previous genetic studies. Thousands of 15 complex traits-associated ncRNAs were catalogued by using previous GWAS findings.

(E) Schematic of pinpoint mutation effects by MENTR, compared with GWAS findings and conventional eQTL findings. 
Figure 2. Cell-type-specific prediction of promoter- and enhancer-level expression by MENTR.

(A) Distribution of measured expression levels for the bins of probability from the trained MENTR ML model trained by CAGE transcriptome of corneal epithelial cell (a

5 representative result). (B) Summary of predictive accuracy (AUROC) for the FANTOM5 347 sample ontologies. Purple boxes show the AUROC for the only promoters including the annotation indicated in x-axis. The box plots show the first and third quartiles, the center line represented the median, the upper whisker extended from the hinge to the highest value that is within $1.5 \times \mathrm{IQR}$ (inter-quartile range) of the hinge, the lower

10 whisker extended from the hinge to the lowest value within $1.5 \times \mathrm{IQR}$ of the hinge, and the data beyond the end of the whiskers were plotted as points. Random accuracy $(0.5)$ was shown as a dashed line.

Figure 3. Accurate MENTR predictions of mutation effects on ncRNA expression

in a cell-type-dependent manner.

Concordance rate (y-axis) of directions between in silico mutation effect $\left(\beta_{\text {mutgen }}\right)$ and effect size from the QTL study $\left(\beta_{Q T L}\right)$ at the threshold of absolute $\beta_{\text {mutgen }}$ (x-axis). $\beta_{Q T L}$ was obtained from LCL CAGE QTL study (Garieri et al., 2017) in (A-C) and from LCL 
caQTL study (Kumasaka et al., 2019) in (D-F). Accuracy for enhancers was shown in (A, D), that for IncRNA promoters was in (B, E), and that for mRNA promoters was in (C, F). These $\beta_{Q T L}$ values were compared with $\beta_{\text {mutgen }}$ values from the MENTR ML model trained by LCL CAGE transcriptome (red line) as well as models trained by FANTOM5

5347 CAGE transcriptomes (heatmap color, indicating transcriptomic correlation (Spearman's p) with LCL CAGE transcriptome). The bluer, the more unmatched comparison of cell-types. Random accuracy (0.5) was shown as a dashed line.

\section{Figure 4. MENTR predictions to explain and prioritize GWAS findings.}

10 (A) Association of in silico mutation effect sizes with GWAS effect sizes. Regression coefficients of mutation effects on GWAS effect size for the indicated complex diseases (Ishigaki et al., 2019) in x-axis. The coefficients were conditioned on MAF of variants in testing samples (East Asian), absolute variant-TSS distance, and dummy variable for promoter or enhancer. The statistically significant results (Bonferroni-corrected level of

15 significance; $\mathrm{P}<0.05 / 42)$ were highlighted $(\mathrm{red})$. The error bars show standard error of regression coefficient. (B) The number of credible loci of IBD (Huang et al., 2017), whose causal variants were predicted as 'permissive' by MENTR in at least one cell-type or tissue. (C) The number of credible loci, predicted by MENTR for the 
indicated type of transcription. AD, Atopic dermatitis; BrCa, Breast cancer; BtCa, Biliary tract cancer; CAD, Coronary artery disease; CeAn, Cerebral aneurysm; CeCa, Cervical cancer; $\mathrm{CHB}$, Chronic hepatitis B; CHC, Chronic hepatitis C; CHF, Congestive heart failure; CoCa, Colorectal cancer; COPD, Chronic obstructive pulmonary disease; DE,

5 Drug eruption; EnCa, Endometrial cancer; EsCa, Esophageal cancer; GaCa, Gastric cancer; GD, Graves' disease; HemMa, Hematological malignancy; HepCa, Hepatocellular carcinoma; ILD, Interstitial lung disease; IS, Ischemic stroke; LuCa, Lung cancer; NS, Nephrotic syndrome; OvCa, Ovarian cancer; PaCa, Pancreatic cancer; PAD, Peripheral artery disease; PD, Periodontal disease; PrCa, Prostate cancer;

10 PTB, Pulmonary tuberculosis; RA, Rheumatoid arthritis; T2D, Type 2 diabetes; UF, Uterine fibroids.

Figure 5. MENTR illuminating a candidate of a causal variant in Crohn's disease by linking variants with transcribed enhancers in relevant cell-types

15 (A) Association plots from Crohn's disease transethnic meta GWAS (Liu et al., 2015) and asthma transethnic meta GWAS results (Demenais et al., 2018). Bayes Factor (BF) was used in the former, and p-value was used in the latter. BF threshold in the paper $\left(\log _{10} \mathrm{BF}=6\right)$ and Genome-wide significant threshold $\left(P=5 \times 10^{-8}\right)$ were shown as a 
dashed line, respectively. (B) Association plot for SMAD3 eQTL from GTEx v7 eQTL studies. Significant eQTL determined by GTEx (qval < 0.05 ) was shown. The minimum p-value for each locus over tissues was used. (C) in silico mutation effects for the indicated transcripts from MENTR ML model for neutrophil. Absolute mutation effect

5 sizes were shown. Permissive threshold of mutation effect $(0.05)$ was shown as a dashed line. SMAD3, representative promoter (p1@SMAD3).

\section{Tables}

Table 1. GWAS traits-associated promoters and enhancers

\begin{tabular}{|c|c|c|c|c|c|c|c|}
\hline \multirow{2}{*}{ Threshold } & \multirow{2}{*}{$\begin{array}{c}\text { \#Tested } \\
\text { pairs }\end{array}$} & \multirow{2}{*}{ \#variants } & \multicolumn{5}{|c|}{ \#CAGE peaks } \\
\hline & & & & promoter & mRNA & IncRNA & enhancer \\
\hline None & $433,072,776$ & 41,223 & 177,734 & $4 \quad 131,982$ & 542,984 & 85,480 & 45,752 \\
\hline Permissive & 197,306 & 17,306 & 32,979 & 25,204 & 24,502 & 3,548 & 7,775 \\
\hline Robust & 15,395 & 1,256 & 2,067 & 1,693 & 1,235 & 168 & 374 \\
\hline
\end{tabular}

10 Threshold None, all of the tested data; Threshold Permissive, analysis results whose absolute in silico mutation effects were $\geq 0.05$; Threshold Robust, analysis results whose absolute in silico mutation effects were $\geq 0.1$; \#Tested pairs, the number of combination of variant, trait, CAGE peak, and MENTR ML models; \#variants, the distinct 
number of tested GWAS variants and variants in LD; \#CAGE peaks, the number of

CAGE promoters and enhancers.

\section{STAR Methods}

\section{LEAD CONTACT AND MATERIALS AVAILABILITY}

Further information and requests for resources and reagents should be directed to and will be fulfilled by the Lead Contact, Chikashi Terao (chikashi.terao@riken.jp).

\section{METHOD DETAILS}

10 MENTR ML models

We designed the MENTR ML models by combining deep convolutional neural networks (from +/- 100-kb genome sequence around TSS to epigenetic features, using publicly available pre-trained DeepSEA Beluga models (Zhou and Troyanskaya, 2015;

Zhou et al., 2018)) and binary classifier using gradient boosting trees (from epigenetic

15 features to transcription probabilities) (Chen and Guestrin, 2016). In the DeepSEA Beluga model, 2,002 epigenetic features in 200-bp bin were predicted from 200-bp +/900-bp genome sequences (Zhou et al., 2018); then we obtained total 2,002,000 
epigenetic features in +/- 100-kb regions $(2,002 \times 1,000$ bins) using Pytorch (v0.4.0) using cuda 9.0. Based on the hypothesis that transcription would be affected by near epigenetic events, we aggregated each type of epigenetic features by using 5 types of exponential transformation depending bin-TSS distance (Zhou et al., 2018). In this

5 transformation, we did not use transcript strand information because expression levels of transcribed enhancer had no strand information. Notably, using strand information rather slightly decreased predictive accuracies (Figure S11). Mathematical representation of the exponential transformation is as follows:

$$
\begin{gathered}
y=\sum_{i} x_{i} \times f\left(a, t_{i}\right) \\
f\left(a, t_{i}\right)=e^{-a \frac{\left|t_{i}\right|}{200}}
\end{gathered}
$$

where $x_{i}$ is an epigenetic feature of bin $i(i=1, \ldots, 1,000), t_{i}$ is the mean distance

10 between bin $i$ and TSS and $a=\{0.01,0.02,0.05,0.10,0.20\}$ is an empirical parameter of 5 types of transformation (Zhou et al., 2018). The aggregated 10,010 epigenetic features $(2,002 \times 5)$ were used for input variables for gradient boosting trees (GBT) implemented in python xgboost library (v0.72.1). After training GBTs with the parameters (Table S13), we performed isotonic regression (Niculescu-Mizil and

15 Caruana, 2012) using valid datasets (see below) by python sklearn (v0.19.1) to calibrate the output values from the GBT models (Figure S12). We considered that the calibration 
would be an important procedure to compare probabilities among different models and to use probability as a threshold for selecting reliable results of in silico mutagenesis. We used the calibrated probabilities for all analysis unless otherwise specified. We noted that MENTR linear $_{M L}$ models with linear penalized logistic regression models using

5 boosting (Bühlmann, 2006; Chen and Guestrin, 2016) were used for Figure S2 and Figure S8 to reduce computational costs in massive screening conditions. We measured the accuracies of MENTR ML models by AUROC by using R package pROC (v1.8).

\section{Datasets for training and testing MENTR ML models}

We obtained representative TSS positions of promoters and inferred midpoint positions of enhancers from FANTOM5 phase 2.5 data files. We called both as TSS unless otherwise specified. We used the 1,829 samples from the major human primary cell-types and tissues and CAGE transcriptomes, and calculated expression levels of

15 CAGE clusters as counts per million and normalized them by relative log expression methods across all the libraries (see the details in Hon et al., 2017). We calculated mean expression levels of a transcript for 347 types of sample ontologies, which is a set of non-redundant cell $(n=173)$ and tissue $(n=174)$. In the training MENTR ML models, 
we considered $>0$ expression levels as "on" and the others as "off". We used 241,794 autosomal CAGE clusters on chromosome 8 as test data (for evaluate predictive accuracy), and $80 \%$ out of the others as train data (for training ML models) and the remaining as valid data (for early stopping and probability calibration). We used

5 FANTOM CAT annotations (Hon et al., 2017) to define mRNA promoters, IncRNA promoters (antisense IncRNA, intergenic IncRNA, divergent IncRNA, and sense intronic IncRNA), and other types of promoters. In the category specific analysis, we excluded CAGE clusters with multiple annotations.

\section{Evaluation of FP predictions using nascent RNA profiling}

We trained ML models using five ENCODE cell lines (HeLa, HepG2, MCF-7, K562, and GM12878) profiled by CAGE, and defined CAGE peaks whose expression level were 0 but predicted probabilities $>0.5$ in test data as FP predictions. We developed two MENTR ML models from 2 replicates of CAGE transcriptome for each

15 cell line and used FP predictions in either ML models in the following analysis. We evaluated the expression levels of FP predictions in expression levels of the nascent elongating RNAs from NET-CAGE samples treated by $2 \mathrm{M}$ urea lysis buffer (Hirabayashi et al., 2019). We noted that the NET-CAGE could capture transcribed 
CAGE peaks before degradation and therefore detect lowly expressed transcripts including enhancers at the same sequence depth. We evaluated enrichment of expressed nascent RNAs in FP predictions by Fisher's Exact Test using Bonferroni correction $(P<(0.05 / 14))$.

\section{in silico mutagenesis using the trained MENTR ML models}

We predicted probability from hg19 sequence with the reference allele and the alternative allele using the trained MENTR ML models and calculated the difference (in silico mutation effect). In the prospective purpose like cataloging mutation effects of

10 GWAS variants, we calculated in silico mutation effects on all possible transcripts whose TSS located within +/- 100-kb surrounding the variant.

\section{Verification of in silico mutagenesis using promoter and enhancer-level eQTL and $\mathrm{CaQTL}$ results} We obtained published LCL CAGE transcriptomes for 154 unrelated European donors (Garieri et al., 2017) and quantified and normalized them as described in the above. We calculated mean expression levels among the LCL from 154 donors and 
used them for training and testing MENTR ML models (LCL MENTR model). Besides, we obtained published 5,376 promoter usage QTL (puQTL) and 110 enhancer activity QTL (eaQTL) results via personal communication (Garieri et al., 2017). We obtained $1 \mathrm{KG}$ variants in LD with the lead SNPs (1KGp3v5 EUR; $\mathrm{r}^{2} \geq 0.7$, window $\left.\leq 500 \mathrm{~kb}\right)$ and

5 performed in silico mutagenesis for the variants on paired transcripts (promoter or enhancer) using the LCL MENTR model for 7,063 variant-promoter pairs and 161 variant-enhancer pairs $( \pm 100 \mathrm{~kb}$ variants surrounding each peak).

Similarly, we obtained published chromatin accessibility QTL (caQTL) results (Kumasaka et al., 2019). We used 297,308 lead caQTL-peak ATAC-seq pairs from

10227,128 ATAC-seq peaks (only autosomal). We searched $\geq 50 \%$ overlapped regions between the ATAC-seq regions and FANTOM5 promoter or enhancer regions, resulting into 20,963 FANTOM5 permissive enhancer-ATAC-seq pairs and 89,199 FANTOM5 promoter-ATAC-seq peaks. We found variants in LD (1KGp3v5 EUR; $r^{2} \geq 0.7$, window $\leq 500 \mathrm{~kb}$ ) and finally 86,724 variant-promoter pairs and 24,726 variant-enhancer

15 pairs $( \pm 100 \mathrm{~kb}$ variants surrounding each peak).

\section{Verification of in silico mutagenesis using gene-level eQTL results}

We performed in silico mutagenesis of eQTL variants catalogued in GTEx v7 (MAF $\geq$ 
0.05 and sample size $\geq 100$ ) using the paired tissues or cell-types in FANTOM5 sample ontologies (Table S5). In order to compare the GTEx gene-level mutation effects with MENTR promoter-level mutation effect, we filtered the promoter-level mutation effects by the robust or permissive threshold value, aggregated the promoter-level predicted

5 probabilities from input sequences with reference or alternative allele based on the FANTOM CAT annotations, and calculated the difference between them (Figure S8).

\section{Making catalog of GWAS-traits-associated transcripts}

We downloaded GWAS associations and their ancestry information from GWAS catalog

10 (r2019-07-12). From the downloaded 143,963 records, we excluded records including variants-interaction (epistasis) results, unknown risk allele, and variant whose P-value $\geq$ $5 \times 10^{-8}$; records without rSID (dbSNP 151); record, and records not SNP or InDels. Finally, 53,186 records remained (autosomal+chrX; SNP+InDel). By using broad ancestral category information, we split the dataset into only EAS study (1,426 variants),

15 only EUR study $(35,823)$, and others $(15,765)$. For only EAS or EUR study, we obtained variants in $\operatorname{LD}\left(R^{2} \geq 0.7\right.$, window $\left.\leq 500 \mathrm{~kb}\right)$ using $1 \mathrm{KGp3}$ datasets for each population. Similarly, we obtained Biobank Japan GWAS variants including lead variants after conditioning analysis from the published papers (Akiyama et al., 2017; Ishigaki et al., 
2019; Kanai et al., 2018) and variants in LD using EAS 1KGp3 datasets.

In the regression analysis in Figure 4, we used all the results obtained in the above in silico mutagenesis, calculated maximum absolute mutation effects (max effects) over the FANTOM5 347 sample ontologies and LCL, and converted the

5 probability-scale values to logit values (logit max effects). We estimated regression coefficients of the logit max effects on GWAS effect size for the indicated complex diseases only using variants $\geq 1 \%$ in each testing dataset. The coefficients were conditioned on MAF of variants, absolute variant-TSS distance, and dummy variable for promoter or enhancer. All the estimates were shown in Table S10.

10

\section{in silico mutagenesis of variants in credible sets from IBD fine-mapping}

We obtained $95 \%$ credible sets in 139 independent associated regions in IBD from the fine-mapping paper (Huang et al., 2017). We calculated in silico mutation effects of the biallelic variants in dbSNP 151. We reported the number of credible

15 regions including variant-transcript pairs with at least permissive mutation effects $(\geq 0.05)$ in Figure 4B, and showed all results of the pairs with robust mutation effects ( $\geq 0.1)$ in Table S11. For the transcript type-specific count in Figure 4C, we only used CAGE peaks whose corresponding transcript pairs were only the indicated transcript 
type.

\section{QUANTIFICATION AND STATISTICAL ANALYSIS}

We used the statistical computing language R (https://www.r-project.org/) in all the

5 statistical testing by the indicated statistical method.

\section{DATA AND CODE AVAILABILITY}

The pre-trained MENTR ML models (347 sample ontologies and LCL) and the source code for training MENTR ML models and running in silico mutagenesis are publicly

10 hosted at https://github.com/koido/XXX (available after acceptance). GWAS trait-associated ncRNA database is publicly available in a user-friendly GUI application (available after acceptance). These are publicly released under GPL v3 and are free for use for academic and non-commercial applications.

\section{Supplemental Information titles and legends}


Figure S1. Details about MENTR ML.

(A) Comparisons between required datasets for MENTR ML models and conventional eQTL study. In MENTR ML models, CAGE transcriptome data is only required. Existing large-scale CAGE transcriptome, such as FANTOM5 datasets, can be used. In eQTL

study, transcriptome data for the tissue and genotypes from the same individuals are required for estimating mutation effects for each transcript $\left(\beta_{\text {eQTL }}\right)$ in a tissue. (B) Workflow of MENTR ML training and evaluation. See the details in METHOD DETAILS section.

10 Figure S2. Accurate prediction of ncRNA expression by combining MENTR ML models with CAGE transcriptome.

(A) Prediction accuracies of ExPecto methods (Zhou et al., 2018) on GTEx RNA sequence datasets (re-analysis of predictive accuracies among 218 types of tissues) and FANTOM5 CAGE transcriptome datasets (347 sample ontologies). (B,C) Prediction

15 accuracies of the indicated methods (x-axis) on IncRNAs (B) and mRNAs (C) in the FANTOM5 CAGE transcriptome datasets. Spearman's $\rho$ values were compared by violin plot and the mean values were shown by dot. P-values were calculated by Wilcoxon signed rank test. 
Figure S3. Maximized predictive accuracy by using $\pm 100-\mathrm{kb}$ sequence.

We compared effects of input genome sequence length ( $x$-axis for each plot), transcript type for training (each violin plot) on predicting promoter- (blue) and enhancer-level

5 expression (red) in FANTOM5 347 sample ontologies. In these analyses, MENTR linear $_{\text {}}$ was used. AUROC values were compared by violin plot and the mean values were shown by dot. P-values were calculated by Wilcoxon signed rank test.

Figure S4. Partial dependency of predictive accuracy on annotation of target

10 promoters.

We compared the dependency of predictive accuracy on the annotation of CAGE transcript. We selected CAGE peaks with each of the indicated annotation and calculated AUROC of them.

15 Figure S5. Illustrative overview of methods to evaluate accuracies of in silico mutation effects.

(A) Comparison between $\beta_{\text {mutgen }}$ and $\beta_{Q T L}$ from LCL CAGE QTL study (Garieri et al., 
2017). (B) Comparison between $\beta_{\text {mutgen }}$ and $\beta_{Q T L}$ from LCL caQTL study (Kumasaka et al., 2019). See the details in METHOD DETAILS section.

Figure S6. Verification of in silico mutation effects on all promoters.

5 Concordance rate ( $y$-axis) of directions of in silico mutation effect $\left(\beta_{\text {mutgen }}\right)$ for all promoters and effect size from the QTL study $\left(\beta_{Q T L}\right)$ at the threshold of absolute $\beta_{\text {mutgen }}$. $\beta_{Q T L}$ was obtained from LCL CAGE QTL study (Garieri et al., 2017) in (A) and from LCL caQTL study (Kumasaka et al., 2019) in (B). See Figure 3.

10 Figure S7. Correlation between in silico mutation effect size and caQTL effect size.

$\beta_{\text {mutgen }}$ (x-axis) was compared with $\beta_{Q T L}$ from LCL caQTL study (Kumasaka et al., 2019), for enhancers in (A) and promoters in (B) In these plots, we assume that $\beta_{Q T L}$ of the lead SNP has the same $\beta_{Q T L}$ of variants in LD. $\rho$, Spearman's $\rho$. red dot, absolute $\beta_{\text {mutgen }} \geq$

150.1 ; triangle dot, absolute variant-TSS distance $>1 \mathrm{~kb}$. We excluded variants with 0 mutation effect from this analysis. 
Figure S8. Gene-level verification of MENTR in silico mutation effects for various types of tissues.

(A) Workflow of calculating gene-level mutation effects $(\Delta y) . \Delta y$ values were calculated from promoter-level mutation effects $\left(\Delta y_{p}\right)$ after filtered by the baseline,

5 permissive, and robust threshold. (B) Concordance rate (y-axis) of directions of the $\Delta y$ and effect size of eQTL from GTEx v7 at the indicated threshold of absolute $\Delta y$. The concordance rates of 26 tissues (Table S5) were shown by violin plot and the mean values were shown as dot. P-values were calculated by Wilcoxon signed rank test.

10 Figure S9. MENTR predictions of causal variants which were experimentally validated.

Association plots (upper panel) and absolute mutation effects (lowered panel) for CCR1 (A) and HLA-DOA (B). The functional variant (rs147398495 and rs381218) and variants in LD ( $r^{2} \geq 0.2$ in 1 KGp3v5 EUR) were shown. The permissive threshold of in silico

15 mutation effect (0.5) was shown as a dashed line. Larger dots mean that the mutation effect sizes are greater than the robust threshold (0.1). The strongest in silico mutation effects among 348 models for each mutation were shown. 
Figure 10. MENTR predictions of causal variants in Crohn's disease by linking known functional variants with transcribed enhancers in relevant cell-types.

in silico mutation effects of rs4456788, rs713875, and their variants in LD. For the association plots, summary statistics of Crohn's disease transethnic meta GWAS from

5 (Liu et al., 2015) were used in (A; for rs4456788) and those from (Franke et al., 2010) were used in (B; for rs713875). eQTL for near-by ICOSLG and LIF were obtained from GTEx v7 eQTL significant results. in silico mutation effects for the indicated transcripts were predicted from the MENTR ML model for CD4 ${ }^{+}, \alpha-\beta$ T cell in (A) and CD8 ${ }^{+}, \alpha-\beta$ T cell in (B), both of which were the cell type with the strongest in silico mutation effects

10 (Table S11). See also Figure 5.

Figure S11. Decreased predictive accuracies in MENTR ML models by using transcript strand information.

We compared effects of considering strand in our MENTR ML models (x-axis, AUROC

15 from ML models using strand information like ExPecto models (Zhou et al., 2018); y-axis, our methods which do not use strand information). We trained ML models by using only promoters. In this figure, MENTR $\mathrm{R}_{\text {linear }}$ was used. 
Figure S12. Calibration of probabilities for accurate metrics.

Representative calibration plots were shown. Percentage of expressed CAGE peaks for each bin were shown in y-axis, and mean values of probabilities for each bin (evenly spaced by 0.2 of range $[0,1])$ were shown in $x$-axis. The number of CAGE peaks in

each bin were shown in the upper bar plots. GBT: only gradient boosting trees; GBT+IR:

isotonic regression after gradient boosting trees, used for MENTR ML models. Sample ontology ID for each plot was UBERON:0010133 in (A), UBERON:0009722 in (B), and UBERON:0005911 in (C).

\section{References}

(DGT), T.F.C. and the R.P. and C., Forrest, A.R.R., Kawaji, H., Rehli, M., Baillie, J.K., Hoon, M.J.L. de, Haberle, V., Lassmann, T., Kulakovskiy, I. V., Lizio, M., et al. (2014). A promoter-level mammalian expression atlas. Nature 507, 462-470.

Aguet, F., Brown, A.A., Castel, S.E., Davis, J.R., He, Y., Jo, B., Mohammadi, P., Park,

15 Y., Parsana, P., Segrè, A. V., et al. (2017). Genetic effects on gene expression across human tissues. Nature 550, 204-213.

Akiyama, M., Okada, Y., Kanai, M., Takahashi, A., Momozawa, Y., Ikeda, M., Iwata, N., 
Ikegawa, S., Hirata, M., Matsuda, K., et al. (2017). Genome-wide association study identifies 112 new loci for body mass index in the Japanese population. Nat. Genet. 49, $1458-1467$.

Alasoo, K., Rodrigues, J., Mukhopadhyay, S., Knights, A.J., Mann, A.L., Kundu, K., Hale,

5 C., Dougan, G., and Gaffney, D.J. (2018). Shared genetic effects on chromatin and gene expression indicate a role for enhancer priming in immune response. Nat. Genet. $50,424-431$.

Andersson, R., Gebhard, C., Miguel-Escalada, I., Hoof, I., Bornholdt, J., Boyd, M., Chen, Y., Zhao, X., Schmidl, C., Suzuki, T., et al. (2014). An atlas of active enhancers across

10 human cell types and tissues. Nature 507, 455-461.

Anish, R., Hossain, M.B., Jacobson, R.H., and Takada, S. (2009). Characterization of

Transcription from TATA-Less Promoters: Identification of a New Core Promoter

Element XCPE2 and Analysis of Factor Requirements. PLoS One 4, e5103.

Ardlie, K.G., Deluca, D.S., Segre, A. V, Sullivan, T.J., Young, T.R., Gelfand, E.T.,

15 Trowbridge, C.A., Maller, J.B., Tukiainen, T., Lek, M., et al. (2015). The

Genotype-Tissue Expression (GTEx) pilot analysis: Multitissue gene regulation in humans. Science (80-. ). 348, 648-660.

van Arensbergen, J., Pagie, L., FitzPatrick, V.D., de Haas, M., Baltissen, M.P., 
Comoglio, F., van der Weide, R.H., Teunissen, H., Võsa, U., Franke, L., et al. (2019).

High-throughput identification of human SNPs affecting regulatory element activity. Nat.

Genet. 51, 1160-1169.

Banovich, N.E., Li, Y.I., Raj, A., Ward, M.C., Greenside, P., Calderon, D., Tung, P.Y.,

5 Burnett, J.E., Myrthil, M., Thomas, S.M., et al. (2018). Impact of regulatory variation

across human iPSCs and differentiated cells. Genome Res. 28, 122-131.

Boyd, M., Thodberg, M., Vitezic, M., Bornholdt, J., Vitting-Seerup, K., Chen, Y., Coskun,

M., Li, Y., Lo, B.Z.S., Klausen, P., et al. (2018). Characterization of the enhancer and

promoter landscape of inflammatory bowel disease from human colon biopsies. Nat.

10 Commun. 9.

Bryois, J., Garrett, M.E., Song, L., Safi, A., Giusti-Rodriguez, P., Johnson, G.D., Shieh,

A.W., Buil, A., Fullard, J.F., Roussos, P., et al. (2018). Evaluation of chromatin

accessibility in prefrontal cortex of individuals with schizophrenia. Nat. Commun. 9,

3121.

15 Bühlmann, P. (2006). Boosting for high-dimensional linear models. Ann. Stat. 34,

$559-583$.

Chen, T., and Guestrin, C. (2016). XGBoost: A scalable tree boosting system. In

Proceedings of the ACM SIGKDD International Conference on Knowledge Discovery 
and Data Mining, (New York, New York, USA: Association for Computing Machinery),

pp. 785-794.

Chen, L., Ge, B., Casale, F.P., Vasquez, L., Kwan, T., Garrido-Martín, D., Watt, S., Yan, Y., Kundu, K., Ecker, S., et al. (2016). Genetic Drivers of Epigenetic and Transcriptional

5 Variation in Human Immune Cells. Cell 167, 1398-1414.e24.

Degner, J.F., Pai, A.A., Pique-Regi, R., Veyrieras, J.-B., Gaffney, D.J., Pickrell, J.K., De Leon, S., Michelini, K., Lewellen, N., Crawford, G.E., et al. (2012). DNase $\square$ I sensitivity

QTLs are a major determinant of human expression variation. Nature 482, 390-394.

Delaneau, O., Zazhytska, M., Borel, C., Giannuzzi, G., Rey, G., Howald, C., Kumar, S.,

10 Ongen, H., Popadin, K., Marbach, D., et al. (2019). Chromatin three-dimensional interactions mediate genetic effects on gene expression. Science 364, eaat8266.

Demenais, F., Margaritte-Jeannin, P., Barnes, K.C., Cookson, W.O.C., Altmüller, J., Ang, W., Barr, R.G., Beaty, T.H., Becker, A.B., Beilby, J., et al. (2018). Multiancestry association study identifies new asthma risk loci that colocalize with immune-cell

15 enhancer marks. Nat. Genet. 50, 42-50.

Donczew, R., and Hahn, S. (2017). Mechanistic Differences in Transcription Initiation at TATA-Less and TATA-Containing Promoters. Mol. Cell. Biol. 38. 
Fahy, J. V. (2009). Eosinophilic and neutrophilic inflammation in asthma insights from clinical studies. In Proceedings of the American Thoracic Society, pp. 256-259.

Farh, K.K.H., Marson, A., Zhu, J., Kleinewietfeld, M., Housley, W.J., Beik, S., Shoresh, N., Whitton, H., Ryan, R.J.H., Shishkin, A.A., et al. (2015). Genetic and epigenetic fine

mapping of causal autoimmune disease variants. Nature 518, 337-343.

Finucane, H.K., Bulik-Sullivan, B., Gusev, A., Trynka, G., Reshef, Y., Loh, P.-R., Anttila, V., Xu, H., Zang, C., Farh, K., et al. (2015). Partitioning heritability by functional annotation using genome-wide association summary statistics. Nat. Genet. 47, $1228-1235$.

10 Finucane, H.K., Reshef, Y.A., Anttila, V., Slowikowski, K., Gusev, A., Byrnes, A., Gazal, S., Loh, P.-R., Lareau, C., Shoresh, N., et al. (2018). Heritability enrichment of specifically expressed genes identifies disease-relevant tissues and cell types. Nat.

Genet. 50, 621-629.

Franke, A., McGovern, D.P.B., Barrett, J.C., Wang, K., Radford-Smith, G.L., Ahmad, T.,

15 Lees, C.W., Balschun, T., Lee, J., Roberts, R., et al. (2010). Genome-wide meta-analysis increases to 71 the number of confirmed Crohn's disease susceptibility loci. Nat. Genet. 42, 1118-1125.

Garieri, M., Delaneau, O., Santoni, F., Fish, R.J., Mull, D., Carninci, P., Dermitzakis, 
E.T., Antonarakis, S.E., and Fort, A. (2017). The effect of genetic variation on promoter usage and enhancer activity. Nat. Commun. 8, 1358.

Gate, R.E., Cheng, C.S., Aiden, A.P., Siba, A., Tabaka, M., Lituiev, D., Machol, I.,

Gordon, M.G., Subramaniam, M., Shamim, M., et al. (2018). Genetic determinants of

5 co-accessible chromatin regions in activated T cells across humans. Nat. Genet. 50,

$1140-1150$.

Hirabayashi, S., Bhagat, S., Matsuki, Y., Takegami, Y., Uehata, T., Kanemaru, A., Itoh, M., Shirakawa, K., Takaori-Kondo, A., Takeuchi, O., et al. (2019). NET-CAGE characterizes the dynamics and topology of human transcribed cis-regulatory elements.

$10 \quad$ Nat. Genet. 51, 1369-1379.

Hoffman, G.E., Bendl, J., Girdhar, K., Schadt, E.E., and Roussos, P. (2019). Functional interpretation of genetic variants using deep learning predicts impact on chromatin accessibility and histone modification. Nucleic Acids Res. 47, 10597-10611.

Hon, C.C., Ramilowski, J.A., Harshbarger, J., Bertin, N., Rackham, O.J.L., Gough, J.,

15 Denisenko, E., Schmeier, S., Poulsen, T.M., Severin, J., et al. (2017). An atlas of human long non-coding RNAs with accurate 5' ends. Nature 543, 199-204.

Huang, H., Fang, M., Jostins, L., Umićević Mirkov, M., Boucher, G., Anderson, C.A., Andersen, V., Cleynen, I., Cortes, A., Crins, F., et al. (2017). Fine-mapping 
inflammatory bowel disease loci to single-variant resolution. Nature 547, 173-178.

Hutchinson, J.N., Raj, T., Fagerness, J., Stahl, E., Viloria, F.T., Gimelbrant, A., Seddon, J., Daly, M., Chess, A., and Plenge, R. (2014). Allele-Specific Methylation Occurs at Genetic Variants Associated with Complex Disease. PLoS One 9, e98464.

5 Imam, T., Park, S., Kaplan, M.H., and Olson, M.R. (2018). Effector T helper cell subsets in inflammatory bowel diseases. Front. Immunol. 9, 1212.

lotchkova, V., Ritchie, G.R.S., Geihs, M., Morganella, S., Min, J.L., Walter, K., Timpson, N.J., Dunham, I., Birney, E., and Soranzo, N. (2019). GARFIELD classifies disease-relevant genomic features through integration of functional annotations with

10 association signals. Nat. Genet. 51, 343-353.

Ishigaki, K., Kochi, Y., Suzuki, A., Tsuchida, Y., Tsuchiya, H., Sumitomo, S., Yamaguchi, K., Nagafuchi, Y., Nakachi, S., Kato, R., et al. (2017). Polygenic burdens on cell-specific pathways underlie the risk of rheumatoid arthritis. Nat. Genet. 49, 1120-1125.

Ishigaki, K., Akiyama, M., Kanai, M., Takahashi, A., Kawakami, E., Sugishita, H.,

15 Sakaue, S., Matoba, N., Low, S.-K., Okada, Y., et al. (2019). Large scale genome-wide association study in a Japanese population identified 45 novel susceptibility loci for 22 diseases. BioRxiv 795948. 
Kadivar, M., Petersson, J., Svensson, L., and Marsal, J. (2016). CD8aß + үס̄ T Cells: A Novel T Cell Subset with a Potential Role in Inflammatory Bowel Disease . J. Immunol. $197,4584-4592$.

Kanai, M., Akiyama, M., Takahashi, A., Matoba, N., Momozawa, Y., Ikeda, M., Iwata, N., 5 Ikegawa, S., Hirata, M., Matsuda, K., et al. (2018). Genetic analysis of quantitative traits in the Japanese population links cell types to complex human diseases. Nat. Genet. 50, 390-400.

Kelley, D.R., Reshef, Y.A., Bileschi, M., Belanger, D., McLean, C.Y., and Snoek, J. (2018). Sequential regulatory activity prediction across chromosomes with convolutional 10 neural networks. Genome Res. 28, 739-750.

Kim, T.-K., Hemberg, M., Gray, J.M., Costa, A.M., Bear, D.M., Wu, J., Harmin, D.A., Laptewicz, M., Barbara-Haley, K., Kuersten, S., et al. (2010). Widespread transcription at neuronal activity-regulated enhancers. Nature $465,182-187$.

Kristjánsdóttir, K., Kwak, Y., Tippens, N.D., Lis, J.T., Kang, H.M., and Kwak, H. (2018).

15 Population-scale study of eRNA transcription reveals bipartite functional enhancer architecture. BioRxiv 426908.

Kumasaka, N., Knights, A.J., and Gaffney, D.J. (2019). High-resolution genetic mapping of putative causal interactions between regions of open chromatin. Nat. Genet. 51, 
$128-137$.

Lamparter, D., Marbach, D., Rueedi, R., Kutalik, Z., and Bergmann, S. (2016). Fast and

Rigorous Computation of Gene and Pathway Scores from SNP-Based Summary

Statistics. PLoS Comput. Biol. 12, 1-20.

5 Liu, J.Z., Van Sommeren, S., Huang, H., Ng, S.C., Alberts, R., Takahashi, A., Ripke, S.,

Lee, J.C., Jostins, L., Shah, T., et al. (2015). Association analyses identify 38

susceptibility loci for inflammatory bowel disease and highlight shared genetic risk

across populations. Nat. Genet. 47, 979-986.

Mattioli, K., Volders, P.-J., Gerhardinger, C., Lee, J.C., Maass, P.G., Melé, M., and Rinn,

10 J.L. (2019). High-throughput functional analysis of IncRNA core promoters elucidates

rules governing tissue specificity. Genome Res. 29, 344-355.

Maurano, M.T., Humbert, R., Rynes, E., Thurman, R.E., Haugen, E., Wang, H.,

Reynolds, A.P., Sandstrom, R., Qu, H., Brody, J., et al. (2012). Systematic Localization

of Common Disease-Associated Variation in Regulatory DNA. Science (80-. ). 337,

$15 \quad 1190-1195$.

Miller, C.L., Pjanic, M., Wang, T., Nguyen, T., Cohain, A., Lee, J.D., Perisic, L., Hedin, U., Kundu, R.K., Majmudar, D., et al. (2016). Integrative functional genomics identifies regulatory mechanisms at coronary artery disease loci. Nat. Commun. 7. 
Murakawa, Y., Yoshihara, M., Kawaji, H., Nishikawa, M., Zayed, H., Suzuki, H.,

FANTOM Consortium, and Hayashizaki, Y. (2016). Enhanced Identification of

Transcriptional Enhancers Provides Mechanistic Insights into Diseases. Trends Genet.

32, 76-88.

5 Niculescu-Mizil, A., and Caruana, R.A. (2012). Obtaining Calibrated Probabilities from

Boosting.

Pelikan, R.C., Kelly, J.A., Fu, Y., Lareau, C.A., Tessneer, K.L., Wiley, G.B., Wiley, M.M.,

Glenn, S.B., Harley, J.B., Guthridge, J.M., et al. (2018). Enhancer histone-QTLs are

enriched on autoimmune risk haplotypes and influence gene expression within

10 chromatin networks. Nat. Commun. 9, 2905.

Saunders, G., Baudis, M., Becker, R., Beltran, S., Béroud, C., Birney, E., Brooksbank,

C., Brunak, S., Van den Bulcke, M., Drysdale, R., et al. (2019). Leveraging European

infrastructures to access 1 million human genomes by 2022. Nat. Rev. Genet. 20,

693-701.

15 Terao, C., Yoshifuji, H., Nakajima, T., Yukawa, N., Matsuda, F., and Mimori, T. (2016).

Ustekinumab as a therapeutic option for Takayasu arteritis: from genetic findings to

clinical application. Scand. J. Rheumatol. 45, 80-82.

Turner, A.W., Martinuk, A., Silva, A., Lau, P., Nikpay, M., Eriksson, P., Folkersen, L., 
Perisic, L., Hedin, U., Soubeyrand, S., et al. (2016). Functional analysis of a novel genome-wide association study signal in SMAD3 that confers protection from coronary artery disease. Arterioscler. Thromb. Vasc. Biol. 36, 972-983.

Wéra, O., Lancellotti, P., and Oury, C. (2016). The Dual Role of Neutrophils in 5 Inflammatory Bowel Diseases. J. Clin. Med. 5, 118.

Yadav, P.K., Chen, C., and Liu, Z. (2011). Potential role of NK cells in the pathogenesis of inflammatory bowel disease. J. Biomed. Biotechnol. 2011, 348530.

Zhang, Z., Lee, J.H., Ruan, H., Ye, Y., Krakowiak, J., Hu, Q., Xiang, Y., Gong, J., Zhou, B., Wang, L., et al. (2019). Transcriptional landscape and clinical utility of enhancer

10 RNAs for eRNA-targeted therapy in cancer. Nat. Commun. 10.

Zhou, J., and Troyanskaya, O.G. (2015). Predicting effects of noncoding variants with deep learning-based sequence model. Nat. Methods 12, 931-934.

Zhou, J., Theesfeld, C.L., Yao, K., Chen, K.M., Wong, A.K., and Troyanskaya, O.G. (2018). Deep learning sequence-based ab initio prediction of variant effects on expression and disease risk. Nat. Genet. 1. 


\section{Figure 1.}

bioRxiv preprint doi: https://doi.org/10.1101/2020.03.29.011205; this version posted March 31, 2020. The copyright holder for this preprint (which was not certified by peer review) is the author/funder. All rights reserved. No reuse allowed without permission.

A
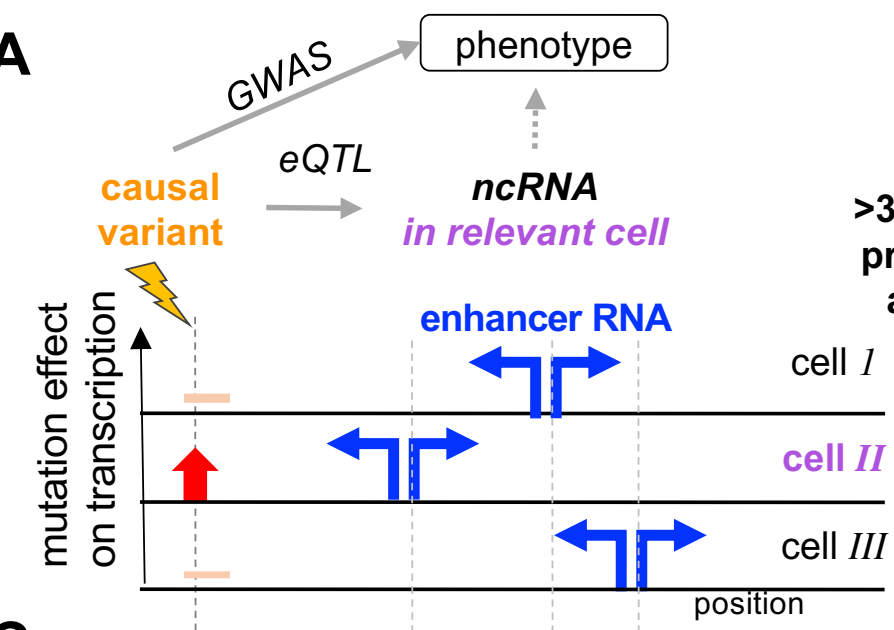

C

...ACAGAAAGCTCGATCGCGCITCGCGA $\cdots$ (hg19 ref. seq)

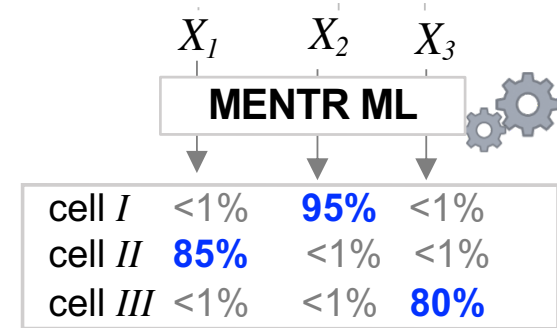

$X_{i}: \pm 100 \mathrm{~kb}$ sequence

expression classifier

using non-linear stacking of epigenetic features

$Y$ : expression probability

in CAGE transcriptome

-ACGGAA $\cdots$ : in silico mutagenesis (of any cis-variant)

\begin{tabular}{|lcll|}
\hline cell $I$ & 0 & 0 & 0 \\
cell $I I$ & $\mathbf{+ 1 0 \%}$ & 0 & 0 \\
cell III & 0 & 0 & 0
\end{tabular}$\quad \Delta Y$ : mutation effect

D

proposing complex traits-associated ncRNAs

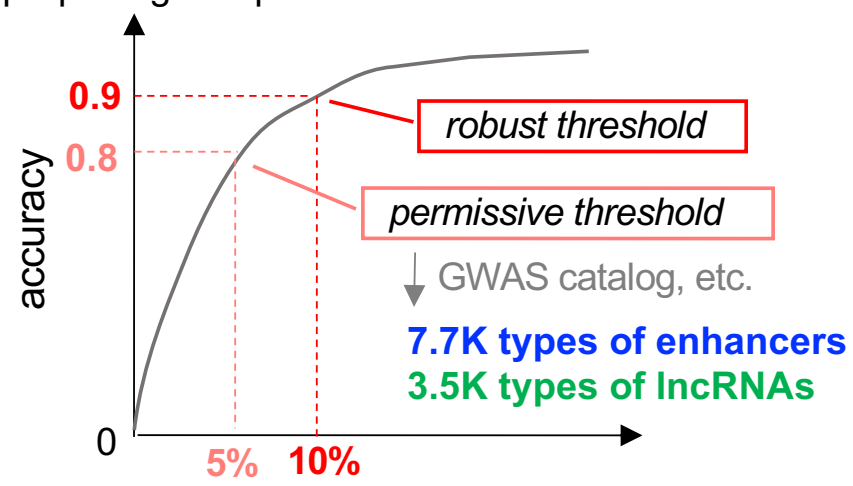

probability change
E discovering causal variant in enhancer regions

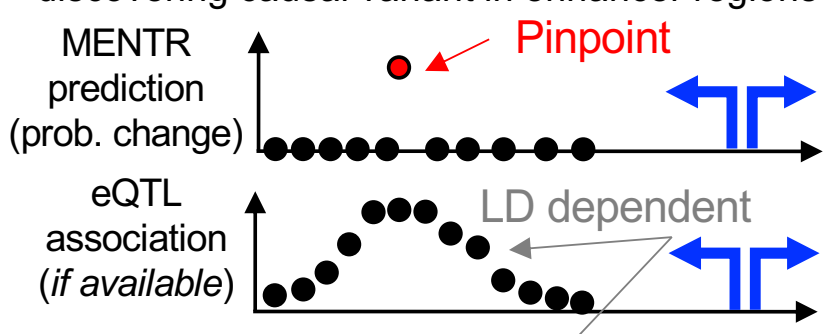

GWAS association

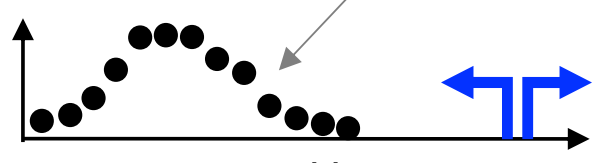

position 
Figure 2.

bioRxiv preprint doi: https://doi.org/10.1101/2020.03.29.011205; this version posted March 31, 2020. The copyright holder for this preprint (which was not certified by peer review) is the author/funder. All rights reserved. No reuse allowed without permission.

A

Corneal epithelial cell

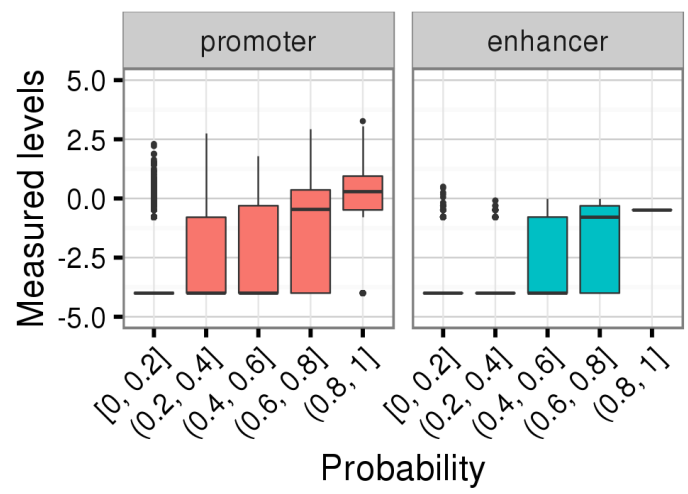

B

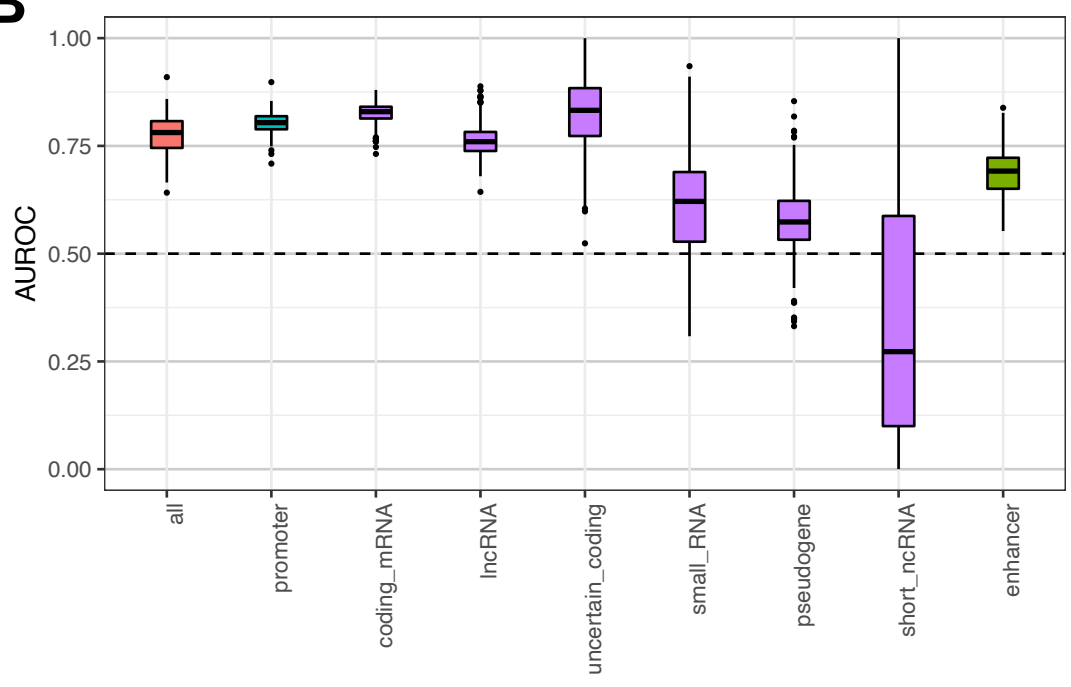

Annotation for CAGE promoter peak 


\section{Figure 3.}

bioRxiv preprint doi: https://doi.org/10.1101/2020.03.29.011205; this version posted March 31, 2020. The copyright holder for this preprint (which was not certified by peer review) is the author/funder. All rights reserved. No reuse allowed without permission.

A

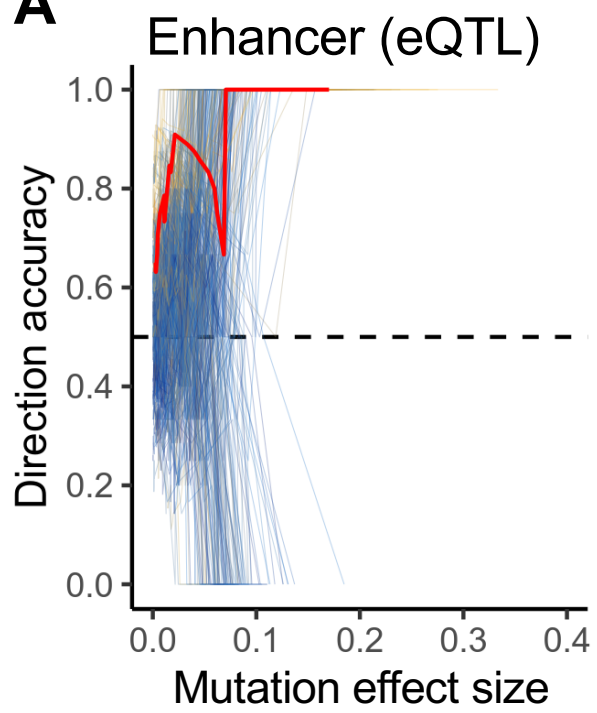

B $\operatorname{IncRNA}(\mathrm{eQTL})$

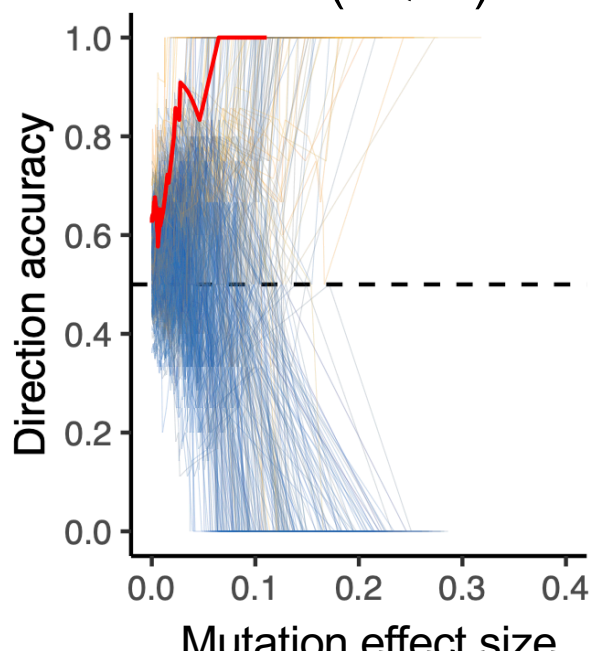

C mRNA (eQTL)

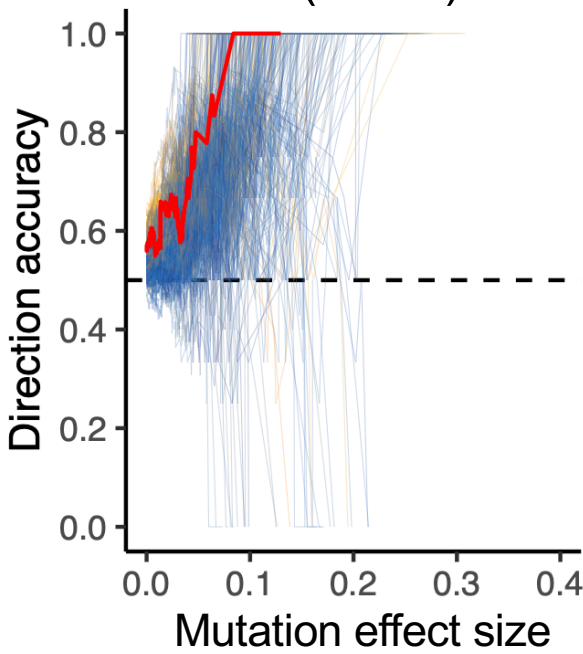

D Enhancer (caQTL)
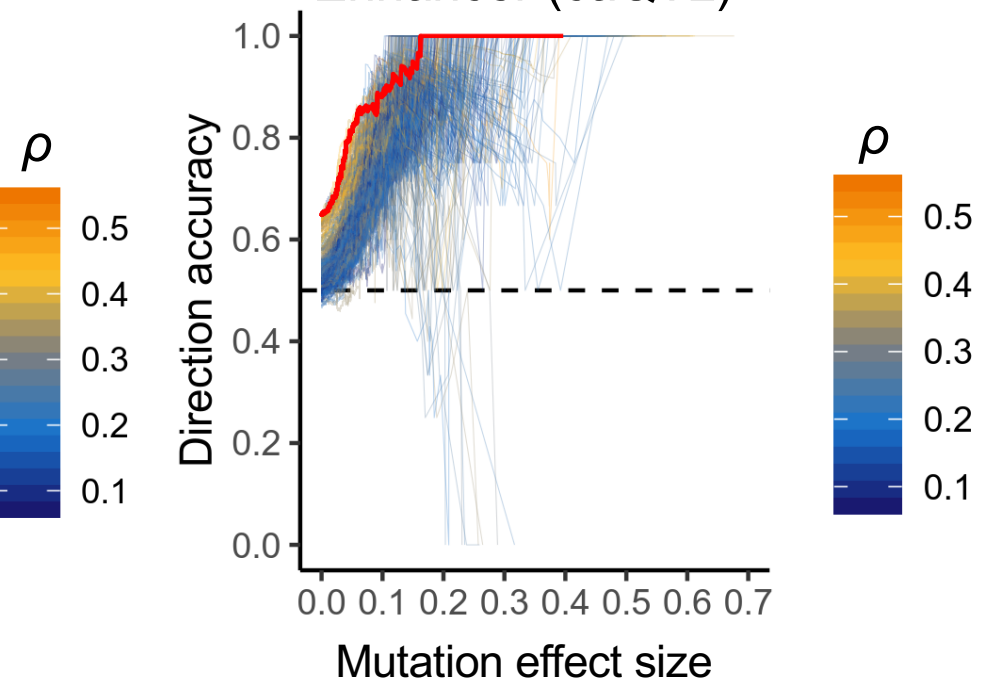

E IncRNA (caQTL)

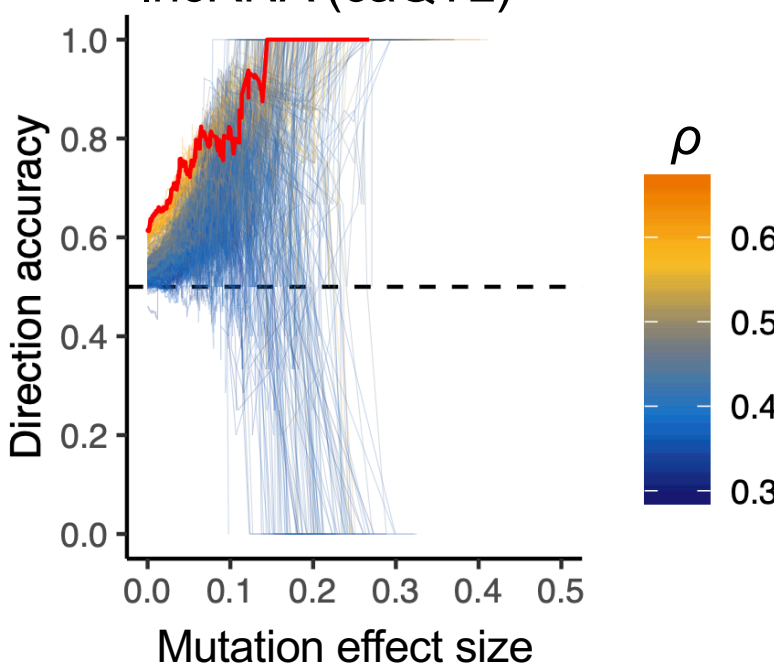

$\mathbf{F}$

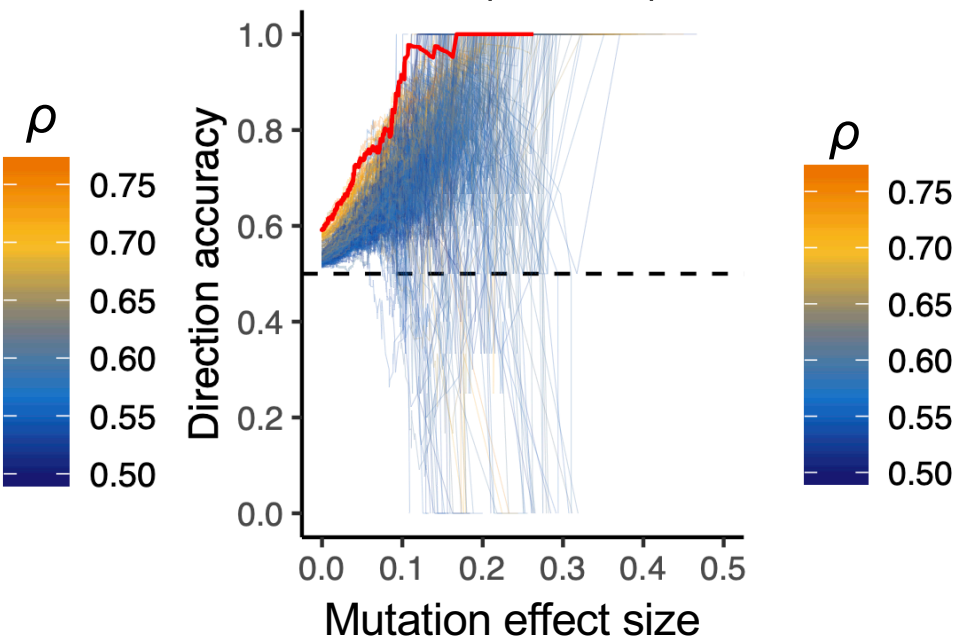




\section{Figure 4.}

bioRxiv preprint doi: https://doi.org/10.1101/2020.03.29.011205; this version posted March 31, 2020. The copyright holder for this preprint (which was not certified by peer review) is the author/funder. All rights reserved. No reuse allowed without permission.

A

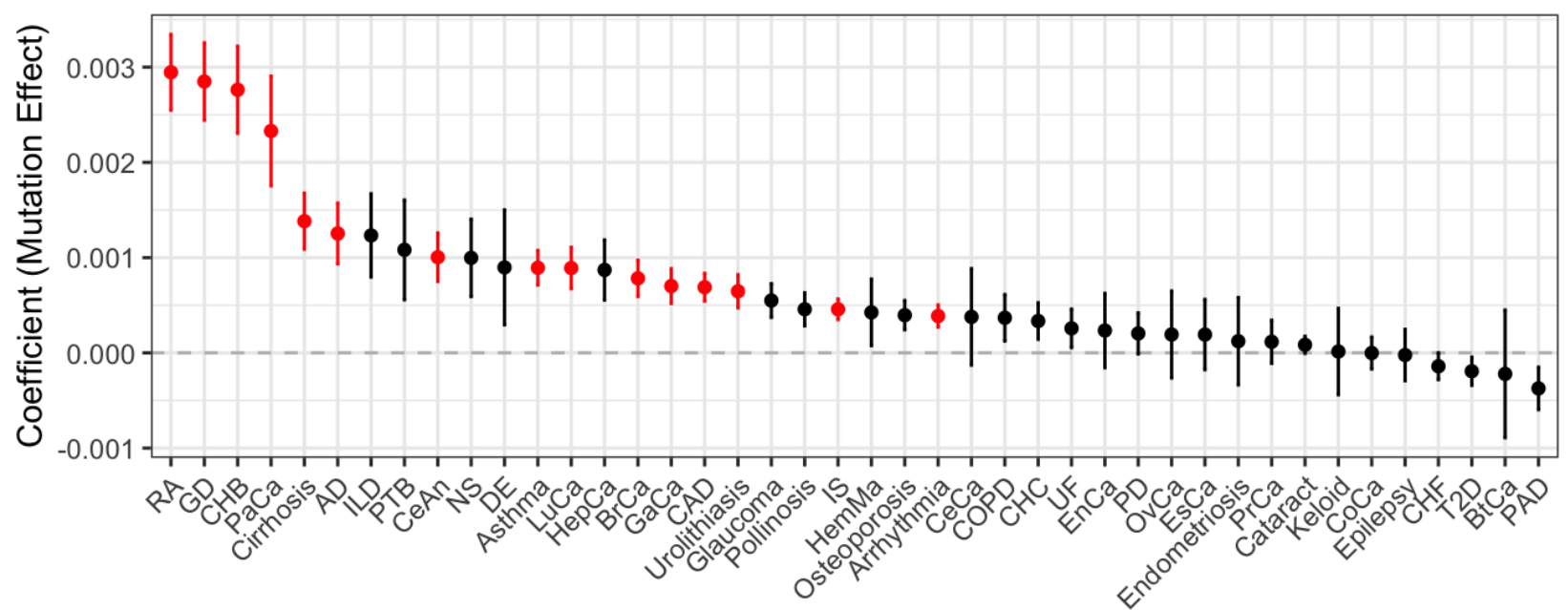

\section{B}

139 independent loci from IBD fine-mapping

(Huang et al., 2017)

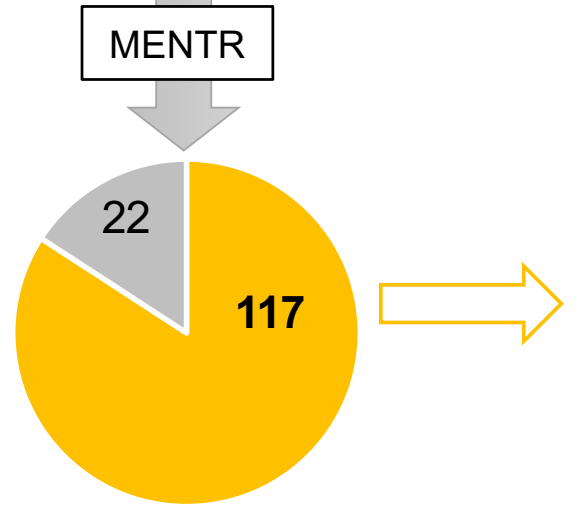

- Predicted by MENTR

Iners

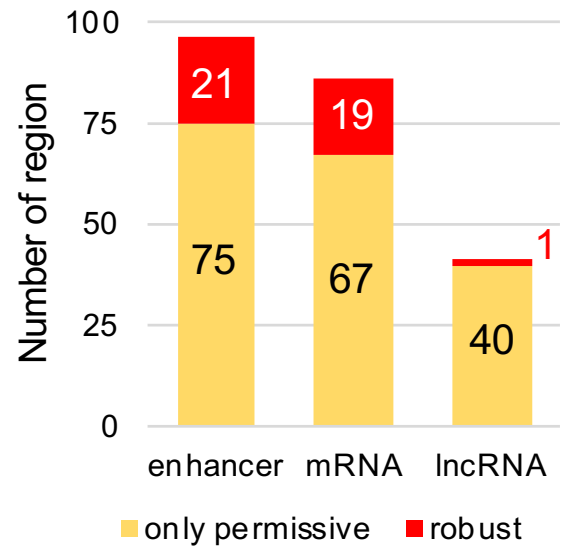


Figure 5.

A

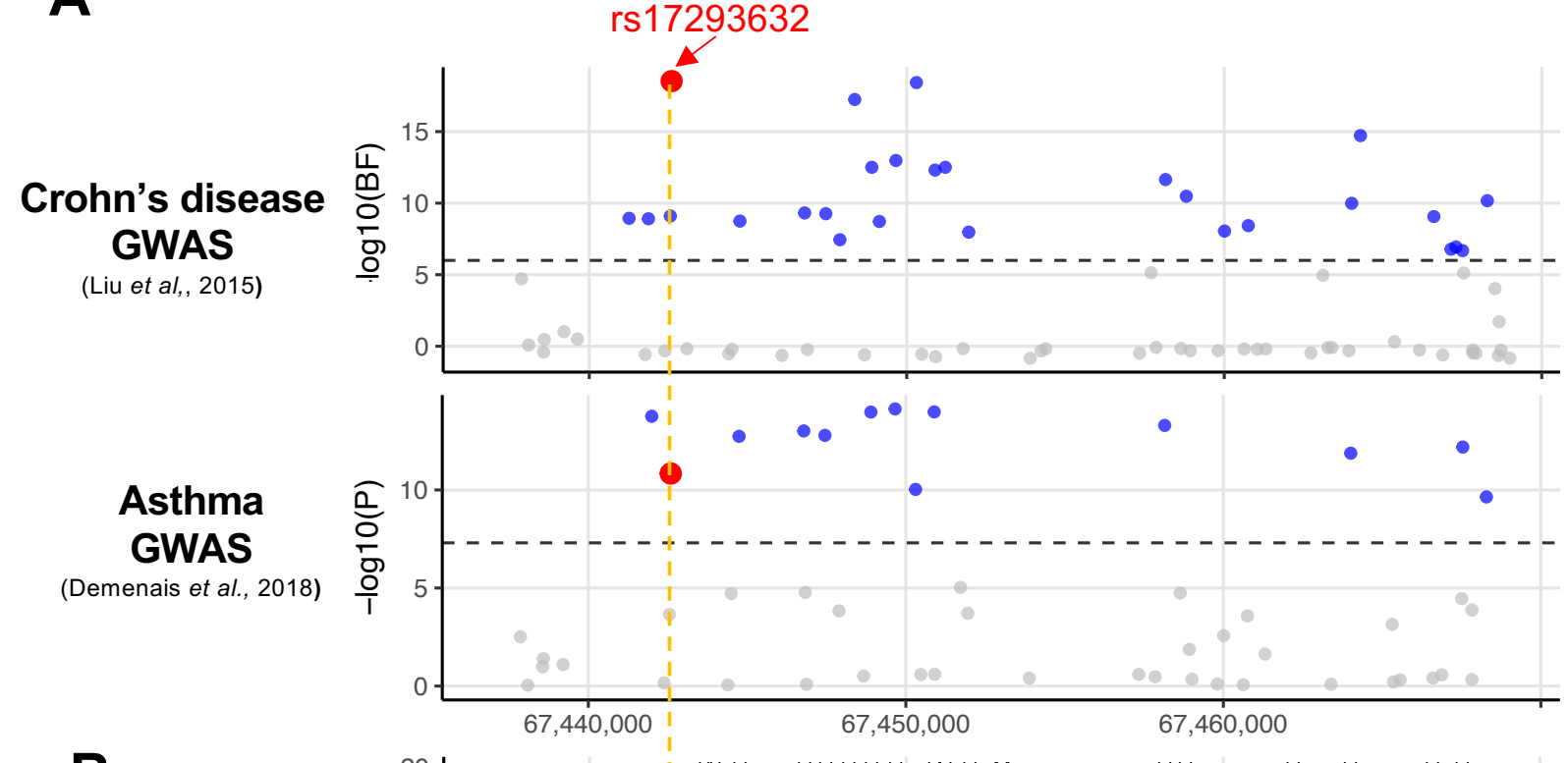

B

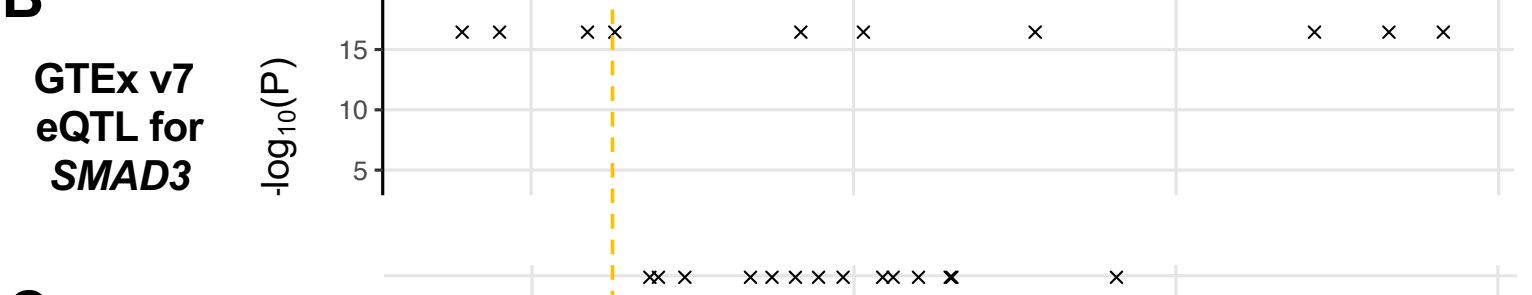

C

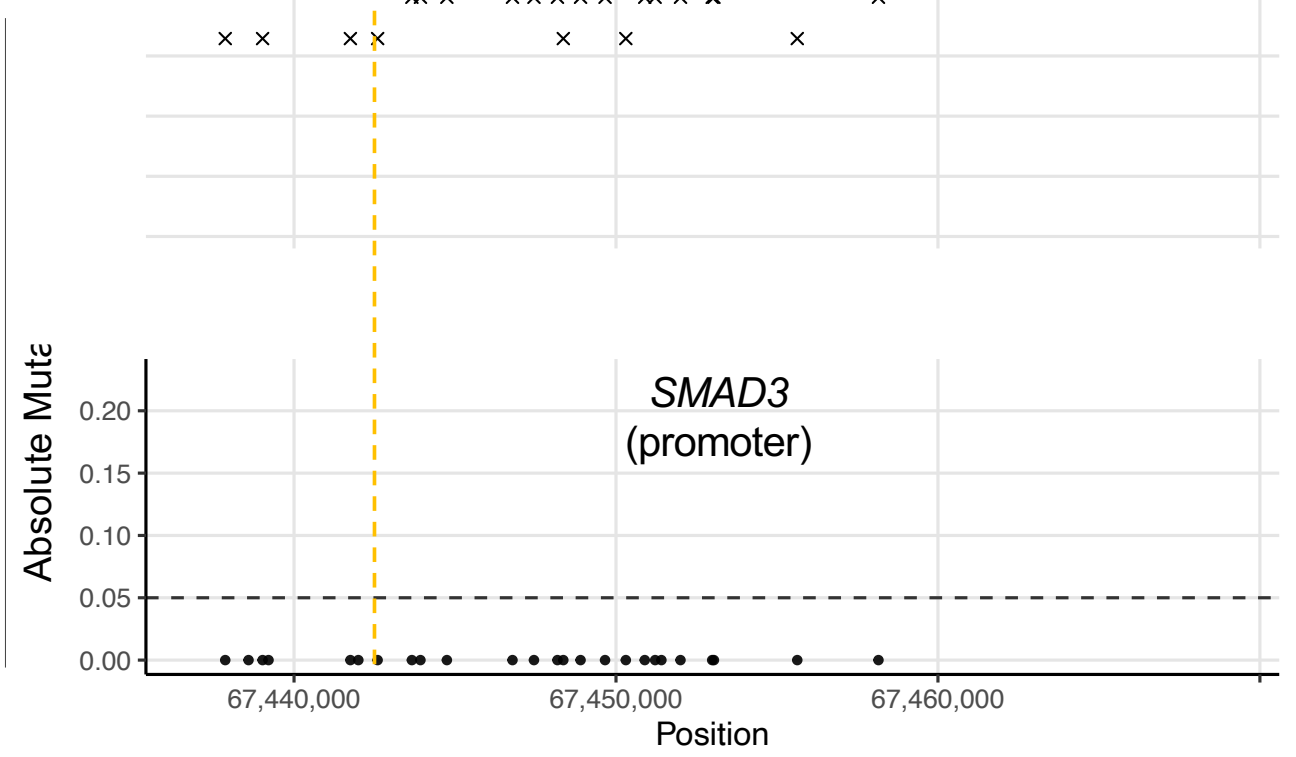

(chr15) 